\title{
De las aulas universitarias a la red. Un proyecto innovador aplicado al aprendizaje y difusión de la literatura catalana barroca
}

Eulàlia Miralles $^{\mathrm{a}}$, Verònica Zaragoza ${ }^{\mathrm{b}}$

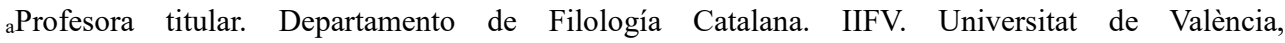
eulalia.miralles@uv.es. b Profesora colaboradora. Universitat Oberta de Catalunya, vzaragozago@uoc.edu.

\begin{abstract}
This paper focuses on the description and analysis of an innovative educational experience in the field of the Baroque Catalan Literature. This experience, which has recently been implemented as a pilot test in universities thanks to the a project of innovative teaching, concentrates on the use of tecnological resources and the development of an initial project of Transmedia Storytelling to foster the motivation of students and generate a more meaningful learning. This case study is grounded in the implementation of the resources and active methodologies of the course on Early Modern Catalan Literature, traditionally based on conventional educational strategies and despised by the historiography. With this goal in mind, we address the theoretical aspects and preliminary goals and explain its developments, to try to reveal the pedagogical benefits that an experience like that one we present here can imply for teaching old literature at the universitarian level.
\end{abstract}

Keywords: Transmedia Storytelling; use of TICs in the educational process; active learning; cooperative learning; project of innovative teaching; higher education; Baroque Catalan Literature.

\footnotetext{
Resumen

Esta comunicación se centrará en la descripción y el análisis de una experiencia docente innovadora en el ámbito de la enseñanza de la literatura catalana barroca. La experiencia, implementada recientemente como actividad piloto en aulas de nivel de enseñanza superior gracias a la concesión de un proyecto de innovación docente, se centra en el uso de los recursos tecnológicos y en el establecimiento de un proyecto de narrativa transmedia inicial, con el fin de favorecer una mayor motivación en los alumnos y revertir en un aprendizaje activo más significativo. Este estudio
} 
De las aulas universitarias a la red. Un proyecto innovador aplicado al aprendizaje y difusión de la literatura catalana barroca

de caso se cimienta en la implementación de los recursos y metodologías activas en la asignatura de Literatura Catalana Moderna, tradicionalmente denostada por la historiografía y basada en entornos educativos convencionales. Para ello, planteamos los aspectos teóricos y objetivos previos a la puesta en marcha del proyecto y explicamos el desarrollo del mismo, para tratar de deslindar los beneficios pedagógicos que una experiencia como la que aqui planteamos puede implicar en la enseñanza de literatura antigua en niveles universitarios.

Palabras clave: narrativa transmedia; uso de las TICs en el proceso de educativo; aprendizaje activo; aprendizaje cooperativo; proyecto de innovación docente; educación superior; literatura catalana barroca.

\section{Introducción: algunas precisiones y delimitación teórica}

El presente trabajo pretende explicar y poner en valor los beneficios pedagógicos de una experiencia de innovación docente aplicada como actividad piloto a la enseñanza universitaria de literatura catalana barroca, la cual se había venido sustentando en metodologías tradicionales de enseñanza-aprendizaje: la lección magistral y el comentario de texto del profesorado en el aula, y el método de evaluación clásico (realización de ejercicios periódicos y examen final), sin atender al gran abanico de posibilidades que las nuevas tecnologías pueden ofrecer al docente.

Como veremos, esta experiencia pretende superar dichos entornos convencionales, centrándose precisamente en la aplicación de las tecnologías de la información y la comunicación (TICs) y, más específicamente, en una propuesta de adaptación de un proyecto colectivo de narrativa transmedia, como elementos que pueden contribuir firmemente al aprendizaje autónomo y a una mayor participación e implicación de las y los estudiantes en la mejora de los procesos de enseñanza-aprendizaje.

En particular, en este estudio planteamos y analizamos los primeros resultados del proyecto de innovación educativa y mejora de la calidad docente concedido por la Universitat de València a la propuesta «De l'aula a la xarxa: literatura catalana del barroc» (UVSFPIE_RMD16-418839), coordinado por Eulàlia Miralles (con formación y experiencia específica en entornos de enseñanza virtual) y adscrito al 'Programa B: Renovación de metodologías docentes', que según convocatoria «persigue experimentar escenarios de ensayo de nuevos instrumentos metodológicos y renovadas técnicas de mejora de los procesos de enseñanza-aprendizaje». En definitiva, con este estudio de caso que aportamos, adaptado a un nivel superior de la enseñanza de la literatura catalana antigua, pretendemos proporcionar nuevos datos al debate sobre los beneficios pedagógicos de la aplicación de recursos tecnológicos de apoyo al aprendizaje (Canós \& Ramón 2005). Más concretamente, queremos mostrar la idoneidad de plantear un proyecto de práctica colectiva de transmedia, explorada con fines pedagógicos con el objetivo de ayudar a los alumnos en la profundización del conocimiento de ciertos autores del período estudiado, desde planteamientos nunca abordados en el campo de la enseñanza de la literatura catalana. En 
ese sentido, no hace falta justificar que la planificación de dicho proyecto y su aplicación a la disciplina de la enseñanza de la literatura venía motivada por la necesidad de flexibilizar los procedimientos educativos para adaptarse a las necesidades que plantea una sociedad como la nuestra, la llamada Sociedad de la Información y el Conocimiento, que debería fundamentarse en sistemas de enseñanzas caracterizados por la «modularidad y la interconexión» (Salinas, 2004).

Por otra parte, en cuanto a la utilidad de la narrativa transmedia planteada en el campo de la innovación educacional (también conceptualizada como transmedia storytelling, crossmedia o narrativas multimedia), más allá de su presencia en los ámbitos del entretenimiento, de la información y de la amplia cultura, ${ }^{1}$ en la última década han sido ampliamente avalados sus usos pedagógicos en contextos de educación secundaria y superior (Ossorio 2014) debido, entre otras cuestiones, a la mayor implicación emocional de los alumnos y al papel claramente autónomo que estos asumen en este tipo de experiencias pilotadas por los mismos, frente al papel orientador y mediador ejercido por el/la docente, alejado del de los entornos educativos tradicionales.

Antes de empezar, debemos precisar que por narrativa transmedia se entiende «Aquella forma de producir contenidos que construye un relato global a través de relatos independientes en diferentes formatos, y cuya convergencia aporta una historia única que se complementa con las historias y características de cada una de las partes que forman el todo. En ella, el público deja de ser un ente pasivo y pasa a interactuar combinando los distintos medios que tiene a su alcance para crear a su manera el discurso y obtener la información que reclama, así como para intercambiar con otros usuarios o consumidores datos, consejos y experiencias basadas en la historia central y en las historias parciales» (Ossorio 2013: 489).

Pese a que ha sido poco explotada como recurso docente en la enseñanza de las literaturas, ${ }^{2}$ no hay duda de que la narrativa transmedia puede abrir la puerta a nuevas posibilidades de aprendizaje en un ámbito tradicionalmente árido y poco atractivo para las y los estudiantes como había sido el de la literatura catalana moderna. Por esta razón, a través de un proyecto de estas características, se conminó al alumnado a explorar y a explotar nuevas plataformas narrativas y medios digitales que les permitiesen crear un contenido unitario relacionado con la asignatura ('la historia global') y a difundirlo a través de los variados canales digitales de los que son usuarios, con resultados positivos tanto a nivel cognitivo como socio-afectivo, como veremos.

1 Aunque no se base en proyectos puramente de aplicación docente, es interesante revisar el espacio 'Narrativa Interactiva' del Grado de Multimedia de la Universitat Oberta de Catalunya $<\underline{\text { http://multimedia.uoc.edu/blogs/narrativa/es/> }}$, con útiles recursos y los trabajos realizados por los estudiantes y profesores [Consulta: 12/01/2017].

2 En este sentido, resulta una experiencia significativa en la difusión de contenidos el proyecto transmedia inspirador Las Sinsombrero: «...un proyecto crossmedia, que utiliza diferentes formatos y plataformas (televisión, internet y publicación), con el objetivo de recuperar, divulgar y perpetuar el legado de las mujeres olvidadas de la primera mitad del siglo XX en España. Desde las figuras femeninas de la Generación del 27 hasta todas aquellas mujeres que con su obra, sus acciones y su valentía fueron y son fundamentales para entender la cultura y la

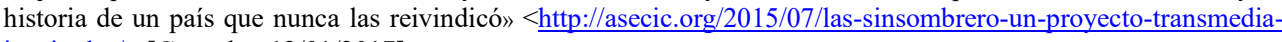
inspirador/> [Consulta: 12/01/2017]. 


\section{Objetivos}

El principal objetivo planteado a las y los estudiantes a través de la experiencia docente que aquí se analiza fue específicamente el diseño y la pretensión de implementación de un proyecto de narrativa transmedia, a través de un uso optimizado de las TICs y los medios combinados (creación de perfiles y cuentas en las redes sociales más influyentes como Facebook, Twitter, representación de obras de teatro, composición de canciones y vídeos grabados para ser reproducidos en medios digitales, hasta la creación de un wikiproyecto, etc.): con ello se les invitaba a confeccionar, a través de múltiples plataformas de expresión y perspectivas, una propuesta de discurso unitario dedicado a dar a conocer a algunos de los principales autores de la literatura catalana moderna, de manera parcelada, pero necesariamente con contenidos interconectados entre sí. Por lo tanto, el objetivo principal del proyecto era que entre todas y todos los alumnos, supervisados por el profesor-guía, se pudiese construir un relato combinado y colectivo sobre el barroco literario, basado en sus principales protagonistas o temas, y que los relatos, creados de manera independiente, convergiesen en la misma historia global.

\subsection{Justificación múltiple del proyecto: profundización y creación de contenidos, mayor índice de participación en las aulas y difusión del período en la red}

Por motivos históricos suficientemente conocidos y explorados, la literatura catalana de la edad moderna ha ocupado un lugar secundario en los planes de estudio de literatura catalana (Rossich 2011). Aunque de unos años a esta parte, la academia ha superado la antinomia Decadència/Renaixença, los estudiantes llegan a la universidad con el prejuicio de la Decadència interiorizado, porque la literatura catalana que estudian en Secundaria y en Bachillerato prolonga, mayoritariamente, la visión de esta etapa como un período literariamente oscuro, decadente y sin excesivo interés; en algunos casos concretos el panorama es incluso peor, puesto que el alumnado ni tan solo es capaz de enumerar algún autor u obra de dicha época. Teniendo en cuenta esta situación, se consideró conveniente y útil explorar en un nivel universitario nuevos procesos experimentales y aplicar ciertos cambios metodológicos que permitiesen a los alumnos adentrarse en un conocimiento más profundo de este período. Se pretendía con ello la creación de un proyecto narrativo innovador, a través de una iniciativa que, de hecho, contribuiría también a la creación de contenidos multimedia contrastados que pudiesen ser relanzados con las nuevas tecnologías, dirigidos no solo al público del ámbito universitario, sino a un público consumidor de contenidos más amplio (que pudiese responder, con una actitud claramente activa). Con ello, el proyecto adquiría un gran interés por partida doble.

Dicho esto, otros de los objetivos concretos que perseguía dicha experiencia docente estaban encaminados a una mayor profundización del aprendizaje y a la dinamización del entorno docente, y se pueden resumir en:

1. Motivar una mayor atención e implicación de los alumnos.

2. Estimular su capacidad de reflexión sobre el período literario. 
3. Generar nuevos roles en el entorno docente y nuevos procesos reflexión que parten del alumno y que se expanden más allá de las clases presenciales, en base al aprendizaje cooperativo y al trabajo colaborativo.

Efectivamente, la experiencia estaba orientada a mejorar la adquisición de habilidades y competencias establecidas en el Documento-Marco sobre la Integración del Sistema Universitario Español en el Espacio Europeo de Educación Superior (EEES), en torno a metodologías docentes dinámicas (rol activo y autónomo en el aprendizaje y en la generación de nuevos conocimientos, desarrollo de la capacidad de reflexión y síntesis...) y colaborativas, pero partía de un modo especial de la voluntad de acercar emocionalmente a los estudiantes del Grado en Filología Catalana a uno de los períodos más desconocidos de la literatura catalana, el de la edad moderna, y, más concretamente, el barroco, con la finalidad de hacerles comprender la vigencia del discurso de los autores barrocos en nuestros días y su utilidad. Por ello, la propuesta pretendía enfatizar el rol activo del estudiante, implicado desde un primer momento, ya fuere identificando los posibles canales de creación y de difusión del discurso (según su capacidad de retroalimentación con el público consumidor de los contenidos, los propios estudiantes u otros usuarios), así como los puntos cardinales de la historia unitaria que debían narrar cada uno de ellos. Sin embargo, el trabajo previo a la planificación fue diseñado y supervisado por los profesores participantes en el proyecto, que aportaron la edición de los textos y las fuentes e información contrastada que permitiese a los estudiantes empezar a crear contenidos, así como las líneas fundamentales que debía seguir el proyecto, según los principios básicos de la transmedia tales como el Worldbuilding o construcción de un mundo unitario (un tema, obra o autor determinado); la serialidad; la multiplicidad; la expansión; la subjetividad...

\section{Desarrollo de la innovación}

\subsection{Diseño previo del proyecto}

Dicho esto, la experiencia docente que aquí se analiza se ha aplicado para el curso 20162017 (1er semestre, septiembre-enero), en los aularios de la Facultat de Filologia, Traducció i Comunicació de la Universitat de València; concretamente con los alumnos de $4^{\circ}$ del Grado de Filología Catalana, en la asignatura de carácter obligatorio "Literatura catalana de l'edat moderna", impartida por dos profesores, que contaba con una matrícula considerable, de 48 estudiantes (para los cuales se presuponía una buena alfabetización en el mundo digital). Antes de abordar las líneas de trabajo, cabría explicar que el grado de Filología Catalana de la Universitat de València cuenta con dos asignaturas que abordan este período en su plan de estudios: en $1^{\circ}$, "Literatura catalana medieval i moderna" y, en $4^{\circ}$, "Literatura catalana de l'edat moderna". Aunque en la asignatura de $1^{\circ}$ se asignan tan solo 3 créditos para el desarrollo de los contenidos sobre los siglos XVI-XVIII, los estudiantes pueden entrar en contacto con la producción literaria del período y se les dota de los instrumentos necesarios básicos (periodización, contexto histórico-cultural, etc.) para que puedan afrontar con garantías de éxito la asignatura de $4^{\circ}$, más monográfica. Ésta, "Literatura catalana de l'edat moderna", de 6 créditos $(3+3)$, combina clases teóricas

2017, Universitat Politècnica de València 5 
expositivas sobre los elementos de construcción del discurso en el renacimiento, barroco e ilustración, y clases prácticas de comentario de textos, desdobladas con la mitad del alumnado, que se busca que sean esencialmente participativas. En definitiva, la metodología propia del aprendizaje convencional, basado en parte en la lección magistral, permite al alumnado acceder a los textos y entenderlos, pero la experiencia docente hasta la fecha venía corroborando que éstos no superaban la distancia existente con la literatura del período: son capaces de diseccionarla pero incapaces de sentirla emocionalmente puesto que la perciben lejana, distante.

\subsection{Implementación del proyecto}

En lo que respecta a la implementación del proyecto, puesto que la asignatura de $4^{\circ}$ es de carácter más monográfico y se basa en una selección forzosa de algunos textos o autores representativos, y asumiendo, además, que el grupo de estudiantes que lo suelen cursar es más reducido, decidimos experimentar una primera fase del proyecto con este grupo, menos masificado, puesto que los niveles educativos superiores apuntan hacia un feedback individual útil menor cuando las dimensiones del grupo es mayor. La duración de dicha asignatura es de un semestre (unas 16 semanas) con cuatro horas de clase presencial a la semana, lo que consideramos que nos debiera permitir alcanzar nuestros objetivos en un plazo de tiempo más prolongado. Por otro lado, por razones prácticas, se optó por acotar cronológicamente el contexto al siglo XVII, concerniente al barroco, el más desarrollado en las clases presenciales.

Así pues, establecido el grupo de trabajo y diseñada la propuesta sobre el siglo XVII, en la primera sesión del curso, se planteó al alumnado la posibilidad de participar de forma voluntaria en una experiencia docente de narrativa transmedia utilizando el instrumento que usan habitualmente para comunicarse y aprender: la red. Para ello, se establecieron adecuadamente los objetivos y premisas de la actividad, planteada solo como complemento de las clases presenciales basadas en las metodologías tradicionales ya comentadas. Se propuso a los alumnos interesados la posibilidad de trabajar voluntariamente en este proyecto novedoso, planteado sin repercusiones en la evaluación de la asignatura. Posteriormente, en las sesiones iniciales, desde posicionamientos del aprendizaje cooperativo, se pidió a los alumnos que reflexionaran sobre los problemas derivados de la enseñanza de un período tradicionalmente minusvalorado y maltratado, con obras prácticamente desconocidas para ellos, como lo es la época moderna. Puesto que el proyecto requería una planificación previa, al delimitar el mensaje global que luego difundirían las y los alumnos, se estableció ésta sobre la base de la necesidad de difundir los autores modernos y sus obras, desde diferentes prismas, pero de manera unitaria.

En segundo lugar, para reparar este desconocimiento, se les pidió que 'inventaran' nuevas formas de transmisión y difusión de la literatura de esta época de forma lúdica, introduciendo en este aspecto la gamificación como herramienta docente para incentivar un aprendizaje activo y entretenido. De hecho, a pesar de que la participación en este proyecto no era una actividad evaluable, se decidió invertir la dotación económica del proyecto en la 
compra de materiales educativos para los alumnos participantes, como premio que les fue comunicado cuando hubo finalizado dicha experiencia docente.

Además, con la voluntad de que se sintieran artífices del proyecto que iban a desarrollar, las instrucciones y directrices que los profesores dieron al alumnado fueron mínimas. En este sentido, consideramos que uno de los aspectos más interesantes de esta iniciativa y que confiere valor al proyecto, de acuerdo con las nuevas líneas de innovación docente, es que convierte al profesor en guía, sin abandonar a los alumnos en este camino de aprendizaje autónomo (Solá, 2004).

Los puntos establecidos previamente con las y los alumnos para el diseño de esta práctica de transmedia fueron:

1. Se establecieron 7 grupos de trabajo conformados por 3-6 estudiantes, que trabajaron de manera coordinada, con un portavoz encargado de coordinar al equipo y de dialogar con el profesor para hacerle llegar las cuestiones y problemas metodológicos o de contenido a los que se enfrentaba el grupo. El profesor tan sólo supervisaba los trabajos de cada grupo y asegura la calidad del contenido de cada proyecto concreto, caso que fuera necesario.

2. Se precisaron las parcelas y temas de trabajo, centrados siempre en un texto barroco en catalán. Para ello, se incentivaron las actividades llevadas a cabo concretamente sobre autores y obras específicamente valencianas aunque podían desarrollar cualquier iniciativa que se ocupara del área lingüística catalana (aparte de Valencia, también de Cataluña, las Islas Baleares, el Rosellón o el Alguer).

3. Para el diseño previo de las diferentes plataformas de la acción transmedia, se invitó a las y los alumnos a imaginar y proponer narrativas para difundir esta literatura en la red, estableciendo previamente la disponibilidad y accesibilidad de varios recursos. Se les concedió libertad en la decisión de los canales y formato de difusión del contenido, de cada parcela o microproyecto, que debían combinarse equilibradamente con una propuesta de contenido acorde a los objetivos establecidos. Y es que cabe tener presente que la narrativa transmedia «disecciona el mensaje en pequeños mensajes independientes, cada uno de los cuales será distribuido después con un formato concreto y en una serie de plataformas concretas con la intención de que sea el receptor quien establezca qué mensajes consumirá para comprender la idea transmitida» (Ossorio 2014: 26-27). Como ejemplo que les pudiese motivar, se les ilustró y motivó con el vídeoclip satírico La vida es sueño de Aldo Narejos (como Calderón de la Barca), Berto Romero (como Miguel Cervantes) y Ana Morgade (como sor Juana Inés de la Cruz), tres personajes mediáticos que reivindican la vigencia, la utilidad y la modernidad del discurso barroco a través de autores paradigmáticos de la literatura española de la época. Lo hacen al son del ritmo musical pegadizo del reggaetón, que busca conectar con los jóvenes a través de una vía que les resulte conocida pero precisamente para denunciar la pérdida de referentes culturales y literarios clásicos 
De las aulas universitarias a la red. Un proyecto innovador aplicado al aprendizaje y difusión de la literatura catalana barroca

y el empobrecimiento musical, léxico y cultural derivados de ciertos productos musicales comerciales de moda.

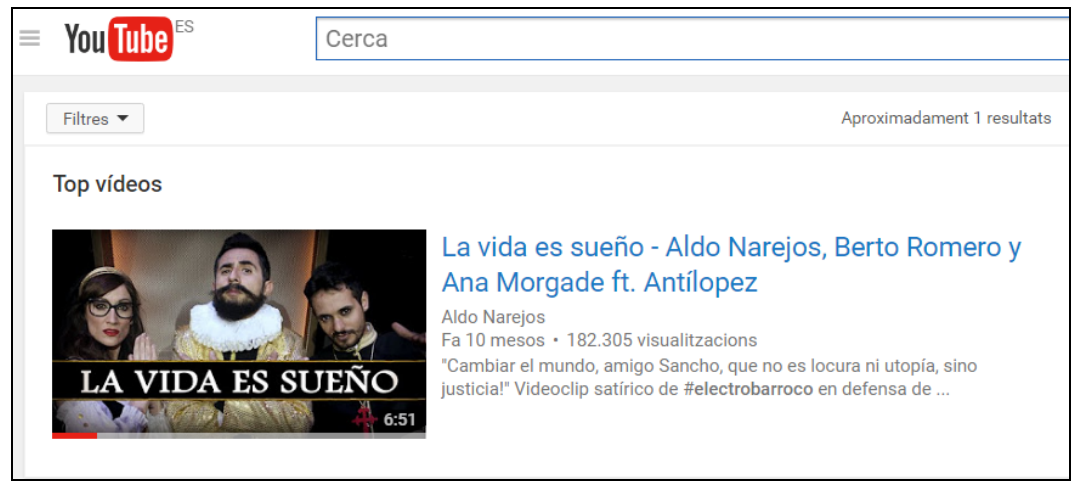

Fig. 1 Video “La Vida es sueño”. Fuente: Youtube

4. Se fijaron como lenguas de difusión de la actividad el catalán, el español y el inglés, de acuerdo con uno de los objetivos complementarios de la experiencia innovadora: la máxima difusión de los contenidos creados por los alumnos y alumnas y la transferencia de conocimientos a la sociedad y visibilidad en entornos más amplios que el universitario.

Establecidas las líneas esenciales del proyecto, y después de dos sesiones presenciales en el aula, se identificaron las limitaciones del alumnado, y el profesorado les dispuso algunas habilidades y herramientas que permitieran superarlas. Se optó, pues, por el coaching educativo, sugiriéndoles a continuación varias propuestas de desarrollo del proyecto, que fuesen complementarias para los diferentes proyectos planteados por ellos. Aunque en su momento no se estableció la actividad explícitamente como un proyecto transmedia, en la etapa de configuración de las propuestas de los canales y contenidos de difusión, se orientó de manera unitaria el trabajo colectivo, estableciendo puntos de contacto entre los diferentes sectores y temas de trabajo para crear una narrativa interactiva unitaria.

\section{Primeros resultados}

La implementación del proyecto transmedia planteado, en un nivel inicial, como complemento de la asignatura "Literatura catalana de l'edat moderna" se ha concretado en varias iniciativas, interactivas entre ellas, que reflejan la participación activa de las y los alumnos y la dinamización de un entorno docente, que va más allá de las aulas, a través de:

1. Creación y actualización de páginas o perfiles en redes sociales como Facebook, centradas en el período en general, como Barroc a la carta (con una descripción que 
explicita los propósitos de la iniciativa: «El Barroc no només té espai als manuals de literatura, i aquesta pàgina vol ser un espai per compartir-lo») $<$ https://www.facebook.com/Barroc-a-la-carta-362885270729896/>, o creadas sobre autores específicos, como el clérigo dietarista valenciano Joaquim Aierdi $<$ https://www.facebook.com/joaquimaierdi/> o el clérigo Franscesc Mulet, al que se ha dedicado una página con teñido carácter escatológico y satírico propio de la época, como sugiere su título Miss Barrocful <https://www.facebook.com/missbarrocful/> -con la descripción significativa: «Miss Barrocful és una iniciativa per fer-vos conéixer un autor del Barroc valencià: el Pare Mulet. Les creadores d'aquesta pàgina formen part de l'estudiantat de Filologia Catalana a la UV i, en concret, de l'assignatura Literatura catalana de l'edat moderna; és per això que s'ha decidit crear aquest compte. Francesc Mulet (16241675), o més conegut com al Pare Mulet, va ser un frare dominic i predicador que va conrear poesia satírica de temàtica escatològica, com és l'obra en què nosaltres ens centrarem: El tractat del pet.»)- y el correspondiente perfil en otra red social de numerosos usuarios: Instagram https://www.instagram.com/missbarrocful/ (imitando el estilo de la más famosa entre los jóvenes marca de artículos de regalo y de frases motivadoras 'Mr. Wonderful'):
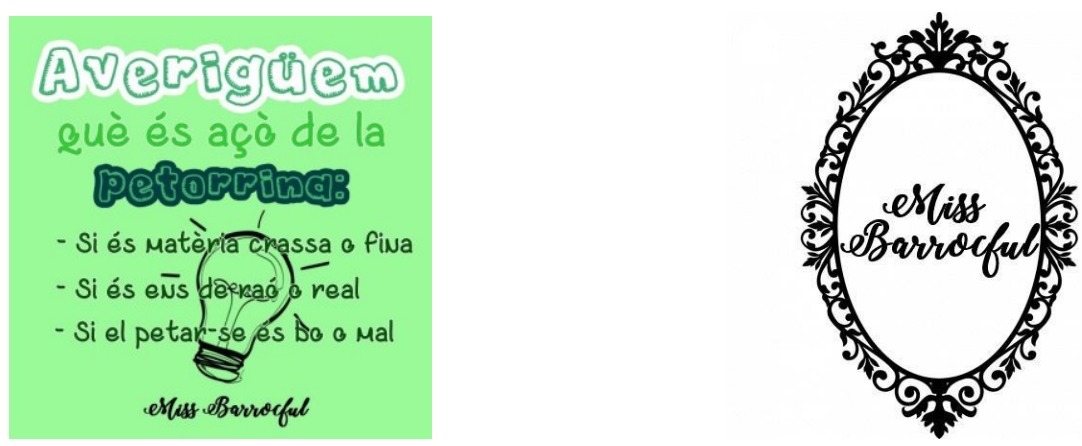

Fig. 2 i 3 Fotografias de la página de facebook "Miss Barrocful" creada por los alumnos en el marco del proyecto transmedia

Como se infiere de estas fotografías, sobre el contenido de las páginas, las y los alumnos mostraron un gran interés en el mantenimiento y actualización de los espacios interactivos, con recreaciones fotográficas acompañadas de transcripciones de fragmentos de obras barrocas, con gran inmersión e implicación de los mismos.

2. Creación y mantenimiento de un blog dedicado a Bernat Català de Valeriola, con entradas con la trascripción de su obra acompañadas de fotografías documentadas $<\underline{\text { http://bernardocataladevaleriola.blogspot.es }}>$ 
De las aulas universitarias a la red. Un proyecto innovador aplicado al aprendizaje y difusión de la literatura catalana barroca

3. Grabación y elaboración de vídeos didácticos, con la participación e implicación de los propios estudiantes en la recopilación, lectura, declamación, representación y canto de los versos de los poetas y dramaturgos del período:

3.1. Vídeo 1: «Romanç que escrigué Fontano embarcat, caminant per lo riu Mossa (Francesc Fontanella)» $<$ https://www.youtube.com/watch?v=yayM4ULeIM\&feature=youtu.be $>$

3.2. Vídeo 2: «Teatre de l'edat moderna: Entremés de la sogra i la nora» $<$ https://www.facebook.com/lia.gutie/videos/10211546699847860/>

3.2. Vídeo 3: «Del Barroc al Present» (sobre uno de los poemas del libro Poesia Festiva, del autor Carles Gasulla d'Ursino) $<$ https://www.youtube.com/watch?v=BbjBzm lpdI\&feature=youtu.be $>$

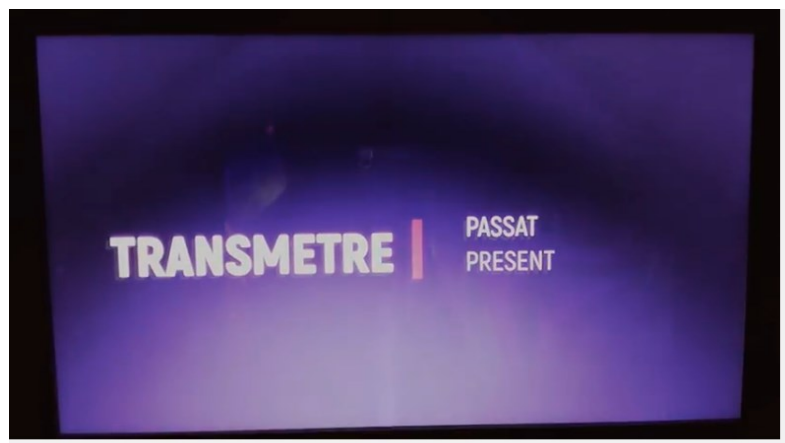

Fig. 4 Vídeo "Del Barroc al Present", elaborado por estudiantes. Fuente: Youtube

\section{Conclusiones}

En este artículo hemos explicado con qué motivación se trató de experimentar con recursos de innovación docente en una asignatura de enseñanza de literatura catalana moderna, tradicionalmente mal conocida por los estudiantes: estos recursos se basan fundamentalmente en el uso de las TICs como apoyo y profundización de los contenidos, y concretamente en el diseño de un proyecto transmedia que permita la difusión de contenidos relacionados con la literatura catalana barroca. Por ello, hemos señalado aspectos teóricos con los que conceptuar la Narrativa Transmedia, para poder aplicarla como recurso de innovación en la enseñanza universitaria. Después de haber delimitado los objetivos y aspectos relacionados con el diseño e implementación del proyecto, hemos explicado los resultados concretos de estas experiencias.

Algunas de las iniciativas llevadas a cabo por los grupos de trabajo en esta prueba piloto de de la Universitat de València aplicada a la asignatura obligatoria "Literatura catalana de l'edat moderna", nos han permitido apuntar algunos de los resultados más destacados 
observados hasta el momento, que muestran los beneficios de la implementación de propuestas de innovación docente como estas en el ámbito de la enseñanza de la literatura, en la línea de lo que han apuntado otros trabajos ${ }^{3}$ :

- la dinamización de la aula y participación voluntaria de la mayoría de las y los estudiantes de la asignatura (32 sobre 48), que han demostrado una mayor implicación, atención y asimilación de los contenidos de la asignatura, a través de la apropiación y recreación de los textos para crear nuevos materiales;

- el avance en el aprendizaje autónomo pero fundamentalmente cooperativo, partiendo del trabajo en grupos, y la estimulación de la cohesión del grupo, mediante actividades externas al entorno docente (elaboración de fotografías en lugares emblemáticos de la ciudad, grabación de vídeos y vídeoclips...);

- el desarrollo complementario del trabajo de las competencias (habilidades comunicativas y de síntesis, selección y citación de la información...) a partir de actividades interactivas entre ellas y totalmente voluntarias;

- la dinamización y la gamificación del entorno educativo, que facilitó además la asunción de roles activos y autónomos de las y los alumnos con el apoyo del profesorado...

En cuanto a las mejoras concretas obtenidas tras la realización de esta actividad docente, los excelentes resultados generales de una prueba de síntesis final sobre la literatura del período nos permitió inferir una mejor comprensión de los contenidos de la asignatura, y una mejor valoración de la importancia de estas interacciones (mayor visibilidad del período, problemas historiográficos en los que siempre se había visto envuelto...), lo cual nos permitió valorar como positiva la implementación de la actividad. Además, efectivamente, uno de los beneficios advertidos en esta experiencia que hemos explicado es que la excelencia educativa, la calidad y las buenas prácticas docentes quedan garantizadas en la medida de que se trata de un proyecto que requiere un diálogo entre profesorado y alumnado que puede contribuir, sin duda, a aumentar el feedback que favorece el aprendizaje.

Por dicho beneficio y otros aspectos apuntados más arriba, consideramos que es interesante seguir las líneas estratégicas implementadas con la concesión de este proyecto de innovación docente, pero ampliando las plataformas de difusión de la literatura catalana barroca, a través de la consolidación de los canales ya creados para ello, pero también con la creación de nuevos medios que permitan consolidar el proceso de innovación docente en

\footnotetext{
${ }^{3}$ Así lo concluyen otros estudios centrados en la aplicación de la narrativa trasnmedia a las aulas universitarias: «La narrativa transmedia es una estrategia de comunicación aplicable al mundo educativo universitario. El hecho de que la narrativa transmedia sea, en resumen, una forma de contar historias o emitir mensajes, permite que sea aplicable al mundo educativo, por cuanto éste supone la transmisión de mensajes cuyo contenido son conocimientos de las distintas áreas del saber. Por ello, las estrategias transmedia aportarían, al igual que lo hacen en los entornos de ficción, información o publicidad, posibilidades de enriquecimiento de los mensajes originales, a la vez que potenciaría la participación de los alumnos y fomentaría la necesidad de reforzar su autosuficiencia y su capacidad crítica, al exigirles seleccionar y elegir el camino a seguir, discriminar información, ampliar lo ofrecido y, en definitiva, convertirse en protagonistas de su propio proceso de aprendizaje.» (Ossorio 2014: 36).
} 
De las aulas universitarias a la red. Un proyecto innovador aplicado al aprendizaje y difusión de la literatura catalana barroca

los aularios de literatura catalana, muy anclados aun en la metodologías y los entornos tradicionales.

Entre los nuevos proyectos que podrían pender de esta experiencia de narrativa transmedia cabría, por ejemplo, la implementación de una página web interactiva, gestionada por el alumnado, que recogiese todo este tipo de iniciativas en la red. Para ello, con el fin de mejorar la experiencia docente, una de las propuestas que consideramos que podrían enriquecer el proyecto, con una mayor participación de las y los estudiantes sería el establecimiento de un nuevo criterio significativo: proponiendo este proyecto como trabajo evaluable de la asignatura, el nombre de estudiantes aumentaría y, por lo tanto, también las iniciativas presentadas.

Vistos los resultados positivos de la experiencia de innovación docente, esta iniciativa deberá ser planteada en un futuro proyecto coordinado entre diferentes universidades que imparten la asignatura, reparando algunas mejoras a introducir, lo que contribuiría a incrementar la implicación activa del alumno en los procesos de aprendizaje, un mayor número de canales de difusión en los que visibilizar nuestras letras barrocas y la creación de una red de estudiantes implicados en una geografía amplia.

\section{Referencias}

CANÓS L. \& RAMÓN F. (2005): “Aplicación de las nuevas tecnologías para el trabajo autónomo del alumno" en Actas del XX Simposium Nacional de la Unión Científica Internacional de Radio. URSI. Gandía: Universitat Politècnica de València, p. 1-4.

Ossorio, M.A. (2013): "Posibilidades de la Narrativa Transmedia aplicada al Periodismo" en F. Ortega, L. Cardeñosa (ed.), Las media enterprises y las industrias culturales. Investigar la comunicación y los nuevos medios, Salamanca: Universidad de $\quad$ Salamanca, pp. $\quad$ 488-497 $<\underline{\text { http://comunicacion3punto0.files.wordpress.com/2013/06/comunicacion3punto0libroactas2012.pdf }>}$ [consulta: 25/03/2017].

OSSORIO, M.A. (2014): “Aplicación de la narrativa transmedia en la enseñanza universitaria en España: Aprendizaje colaborativo, multiplataforma y multiformato". Revista Internacional de Tecnología, Ciencia y Sociedad. vol. 3, núm. 2, < http://tecnociencia-sociedad.com $>$ [republicado de Revista Internacional de Tecnología, Conocimiento y Sociedad 3(2), 2014 (pp. 25-38)] [consulta: 25/03/2017].

Rossich, A. (2011). "La literatura catalana de l'edat moderna: historiografia i crítica" en Panorama crític de la literatura catalana. Edat moderna. Barcelona: Vicens Vives, 17-42.

SALINAS, J. (2004). "Innovación docente y uso de las TIC en la enseñanza universitaria”. Revista de Universidad y Sociedad del Conocimiento (RUSC), vol. 1, n $\mathrm{n}^{\mathrm{o}}$ 1. UOC. <http://www.uoc.edu/rusc/dt/esp/salinas1104.pdf $>$ [Consulta: 10/01/2017].

SolÀ, M. (2004). "La formación del profesorado en el contexto del Espacio Europeo de Educación Superior Avances alternativos". Revista Interuniversitaria de Formación del Profesorado, vol. 18, núm. 3, p. 91-105. 